\title{
Referenzprozesse empirisch bestimmen - Von Vielfalt zu Standards
}

Die Finanz Informatik ist der IT-Dienstleister der Sparkassen-Finanzgruppe. Mit OSPlus stellt das Unternehmen ein Bankensystem zur Verfügung, das alle Kernprozesse unterstützt. Zu den Kunden der Finanz Informatik zählen Sparkassen, Landesbanken, Landesbausparkassen sowie weitere Institute der Sparkassen-Finanzgruppe und der Finanzdienstleistungsbranche. Mit 131 Mio. betreuten Konten und 73 Mrd. abgewickelten technischen Transaktionen pro Jahr hat OSPlus eine Marktdurchdringung von rund der Hälfte des deutschen Retail-Banking-Markts.

Von Prof. Dr. Stephan Aier, Dr. Michael Fichter, Christian Fischer

OSPlus basiert auf einer serviceorientierten Architektur. Bankfachliche Prozesse können durch die Auswahl und Konfiguration von Aktivitätstypen (fachlichen Services) zusammengestellt werden. Um den OSPlus-Anwenderinstituten die Gestaltung ihrer Prozesse zu ermöglichen, können die Fachanwender aus den Instituten diese Prozesse konfigurieren. Ein wesentlicher Erfolgsfaktor der Finanz Informatik besteht darin, dass sie die Unterschiedlichkeit und Individualität der über 400 Institute mit einem einzigen Bankensystem bedienen kann.

Die OSPlus-Anwenderinstitute können die seitens der Finanz Informatik bereitgestellten Standardprozesse direkt nutzen oder diese bei Bedarf an die individuellen Anforderungen anpassen. Der „Preis“ der Institute für die Anpassung an ihre individuellen Anforderungen ist der damit verbundene Konfigurationsaufwand: Obwohl sich Prozesse in OSPlus schnell und einfach konfigurieren lassen, müssen innerhalb der Institute diverse Abstimmungen erfolgen, bevor ein individuell angepasster Prozess operativ eingesetzt werden kann.

In Interviews mit Mitarbeitern der Institute, welche die Universität St.Gallen zusammen mit der Finanz Informatik durchgeführt hat, wurde deutlich, dass die individuelle Prozessgestaltung auch kritisch gesehen wird. Insbesondere kleine und mittelgroße Sparkassen begrüßen zwar die Möglichkeit, Prozesse individuell anpassen zu können, scheuen aber oft den Administrationsaufwand.

\section{Referenzprozesse ermöglichen es, den Administrationsaufwand zu reduzieren}

Eine Möglichkeit, den Administrationsaufwand zu reduzieren, stellen Referenzmodelle dar. Referenzmodelle werden durch den Deutschen Sparkassen- und Giroverband e. V. sowie durch die Regionalverbände der Sparkassen-Finanzgruppe entwickelt. Das definierte Referenzmodell wird im OSPlus durch die Finanz Informatik als Standardprozess bereitgestellt, den die Institute ohne weiteren Administrationsaufwand einsetzen können. Dabei zeigt sich allerdings, dass viele Sparkassen den ausgelieferten Standardprozess anpassen. In Interviews äußerten Sparkassenvertreter einerseits, dass die Referenzprozesse der Regionalverbände zwar wichtig sind, um einen Überblick über den Prozess und seine fachlichen Inhalte zu erhalten. Andererseits aber sei eine Einszu-eins-Umsetzung der Referenzprozesse häufig wenig praktikabel.

Um zu verstehen, an welchen Stellen die Sparkassen einen Prozess anpassen, haben wir exemplarisch den Prozess Personenneuanlage genauer untersucht. Die Prozessmodelle dieses Prozesses liegen im OSPlus als xml-Modelle vor, anhand derer der Prozess im OSPlus ausgeführt wird. Ein Beispiel für eine Ausprägung des Prozesses Personenneuanlage ist in Abbildung 1 dargestellt. 


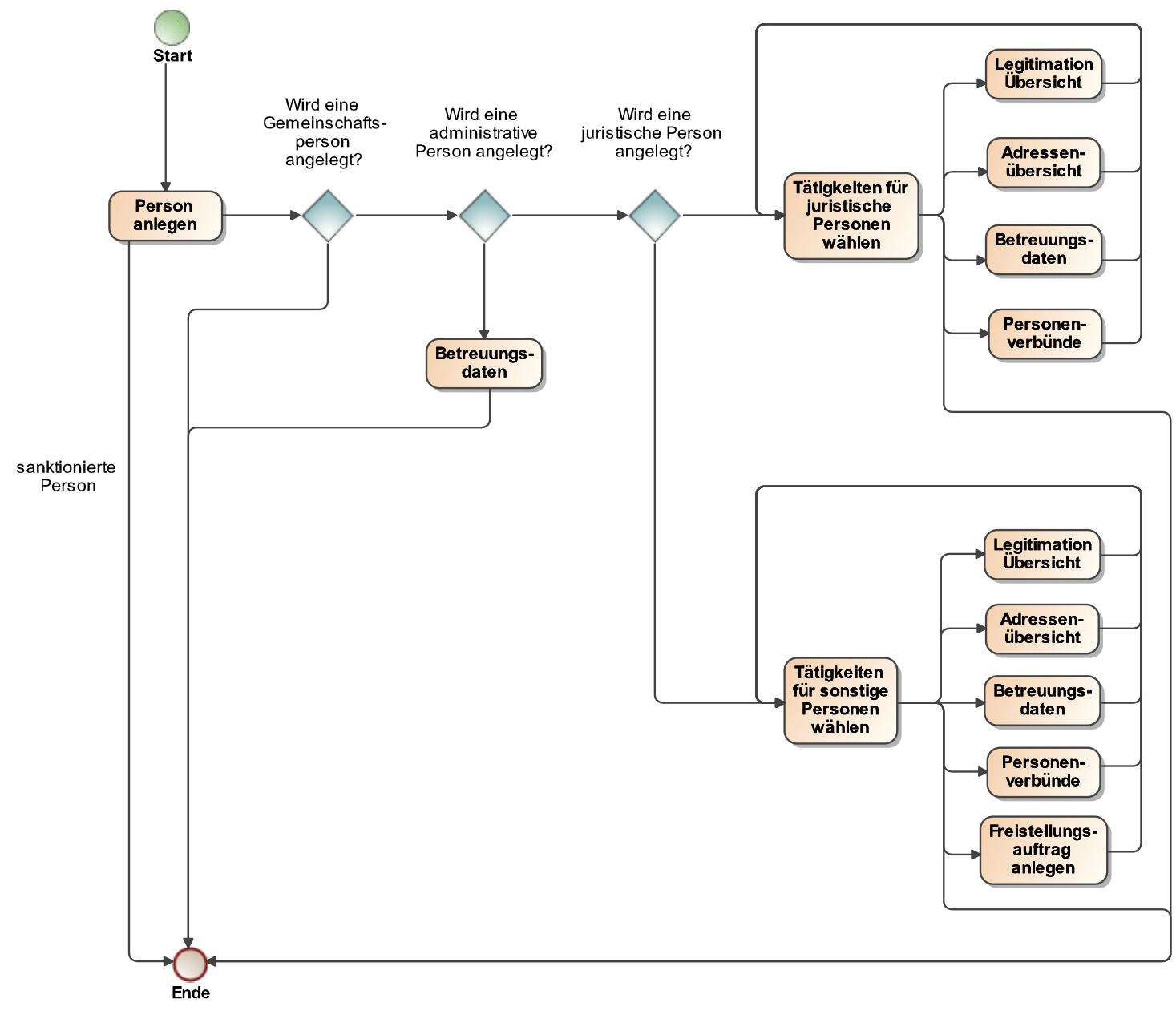

\section{Abbildung 1: Beispiel für eine Ausprägung des Geschäftsprozesses Personenneuanlage}

Eine erste Analyse ergab, dass die von den Instituten eingesetzten Prozesse zur Personenneuanlage eine große Heterogenität aufweisen. Die Institute haben die Prozesse nach ihren jeweils eigenen Kriterien optimiert - und diese Kriterien unterscheiden sich zwischen den Instituten. Die Heterogenität macht deutlich, dass zumindest in diesem Beispiel ein einzelner Referenzprozess nicht dazu geeignet scheint, die unterschiedlichen Anforderungen der Institute abzudecken.

Dieses Problem wird in der Literatur unter dem Stichwort Referenzmodellierungsdilemma diskutiert (Delfmann, 2006). Das Dilemma beschreibt den Trade-off zwischen der Allgemeingültigkeit eines Referenzmodells und seiner Vermarktung. Ein Referenzmodell mit hoher Allgemeingültigkeit kann für viele Unternehmen eingesetzt werden; allerdings muss ein entsprechender Aufwand betrieben werden, um das allgemeine Modell an die spezifischen Rahmenbedingungen eines Unternehmens anzupassen. Im Gegensatz dazu ist der Anpassungsaufwand für ein spezifisches Referenzmodell geringer, es lässt sich aber in nur wenigen Unternehmen einsetzen. Winter (2011) beschreibt diesen Trade-off mithilfe eines Baumdiagramms (vgl. Abbildung 2). Die Wurzel des Baums stellt ein sehr allgemeines Referenzprozessmodell dar; in den Blättern des Baums finden sich spezifische Prozessmodelle, die genau auf die individuellen Anforderungen einzelner Unternehmen abgestimmt sind. Während die Prozessmodelle in den Blättern nicht mehr angepasst werden müssen, hat das allgemeine Referenzmodell in der Wurzel einen sehr hohen Anpassungsaufwand. Die Knoten zwischen der Wurzel und den Blättern stellen weniger allgemeingültige Referenzmodelle dar, deren Anpassungsaufwand zwar geringer ist als der des allgemeinen Referenzmodells, die aber im Gegenzug für weniger Fälle eingesetzt werden können. 


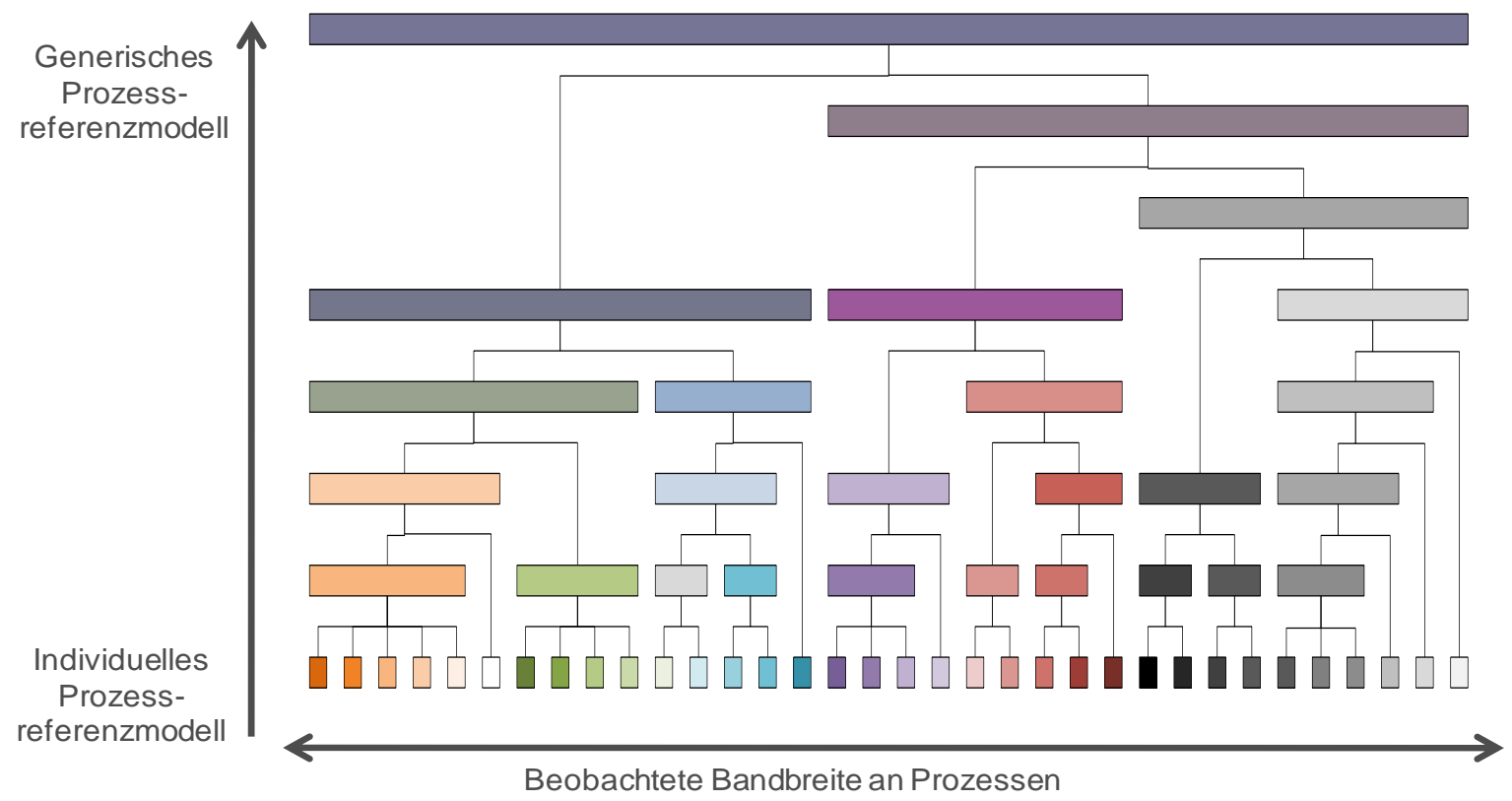

\section{Abbildung 2: Trade-off zwischen Allgemeingültigkeit und Individualität (Winter, 2011)}

\section{Entwicklung eines Clusterverfahrens für Prozesse in OSPlus}

Bei den Verbänden und der Finanz Informatik stellte sich daher die Frage, ob die bei den Instituten angepassten und eingesetzten Prozessvarianten genutzt werden können, um die Referenzmodelle praxisorientiert weiterentwickeln zu können. Da gegebenenfalls auch mehrere Referenzmodelle identifiziert werden können, sollen die Prozesse, die durch die Institute eingesetzt werden, mit Hilfe eines statistischen Clusterverfahren zu Clustern, d.h. Klassen, zusammengefasst werden. Ziel des Clusterverfahrens ist es, Cluster von Prozessen zu bilden, die in sich möglichst homogen und untereinander möglichst heterogen sind. Die Zentren der Cluster sind die natürlichen Kandidaten für die Festlegung neuer Referenzprozesse.

Um ein Verfahren zur Clusterung und einer darauf aufbauenden Ermittlung von Referenzmodellen zu entwickeln, sind wir in drei Schritten vorgegangen:

1. Codierung der Prozesse (Definition einer Abbildung von Prozessen auf eine mathematisch auswertbare Struktur)

2. Definition eines Ähnlichkeitsmaßes zwischen den Codierungen zweier Prozesse

3. Definition eines Clusterverfahrens

Die untersuchten Geschäftsprozesse sind über die in OSPlus integrierte Geschäftsprozesssteuerung modelliert worden. Daher liegen die Prozessmodelle in einem einheitlichen xml-Format vor und die Codierung kann auf einfache und vor allem automatisierbare Weise durchgeführt werden. Ein zusätzlicher manueller Aufwand für eine Prozesserhebung bei den Instituten ist nicht erforderlich.

\section{Codierung}

Zunächst mussten die Prozesse so codiert werden, dass die Berechnung einer Ähnlichkeit zwischen zwei Prozessen möglich ist. Da für Vektoren vielfältige Ähnlichkeitsmaße in der Statistik definiert sind, ist es naheliegend, die relevanten Informationen eines Prozesses auf einen Vektor oder mehrere Vektoren abzubilden. Jung \& Bae (2006) schlagen dazu drei Möglichkeiten vor:

(a) Activity Similarity Measure: Die Berechnung der Ähnlichkeiten zwischen zwei Prozessen basiert auf dem Vorhandensein bzw. Nichtvorhandensein gleicher Aktivitäten in dem Prozess.

(b) Transition Similarity Measure: Die Berechnung der Ähnlichkeit basiert darauf, ob gleiche Aktivitäten in einer direkten Vorgänger-Nachfolger-Beziehung zueinander stehen.

(c) Inferential Transition Similarity Measure: Die Berechnung der Ähnlichkeit zwischen zwei Prozessen basiert nicht nur auf gleichen direkten Vorgänger-Nachfolger-Beziehungen, sondern es werden auch indirekte Vorgänger-NachfolgerBeziehungen berücksichtigt. Je indirekter eine Vorgänger-Nachfolger-Beziehung ist, umso geringer wird sie gewichtet.

Möglichkeit (a) eignet sich insbesondere, um grundlegende fachliche Unterschiede zwischen zwei Prozessen zu identifizieren. Möglichkeiten (b) und (c) berücksichtigen die Ablaufreihenfolge. Jung \& Bae empfehlen, die Prozessähnlichkeit auf Basis von (a) 
und (c) zu berechnen und in einer Konvexkombination zusammenzuführen. Allerdings ergeben sich Schwierigkeiten bei der konkreten Umsetzung der von Jung \& Bae vorgeschlagenen Codierung. Insbesondere die Anforderung, dass die Prozesse keine Zyklen (Loops) enthalten dürfen, verhindert die direkte Nutzung des Inferential Transition Similarity Measure.

Die Finanz Informatik befindet sich in einer sehr guten Ausgangslage für die Berechnung von Ähnlichkeiten zwischen Prozessen in OSPlus. Alle Aktivitäten in einem Prozess sind Instanziierungen von vordefinierten Aktivitätstypen, die für alle Institute einheitlich festgelegt sind. Die fachliche Semantik der einzelnen Prozesse ist in starkem Maße durch ihre Aktivitätstypen festgelegt. Wir können daher Ähnlichkeitsmessungen auf Basis dieser Aktivitätstypen durchführen, die eine Vergleichbarkeit der einzelnen Prozesse untereinander erlauben.

Da die von Jung \& Bae vorgeschlagene Codierung (c) zyklenfreie Prozesse voraussetzt, konnte der dort dargestellte Vorschlag nicht direkt übernommen werden. Wir haben daher die Grundidee weiterentwickelt und uns für drei Codierungen entschieden, welche den Ideen von Jung \& Bae sehr nahe kommen, aber auch für Prozesse mit Zyklen anwendbar sind. Anstelle der Codierung (c) von Jung \& Bae liegt unseren Überlegungen die transitive Hülle der zugehörigen Graphen zugrunde (c*). Im Unterschied zu der von Jung \& Bae vorgeschlagenen Codierung werden in diesem Fall jedoch indirekte Nachfolgerbeziehungen und direkte Nachfolgebeziehungen gleich gewichtet.

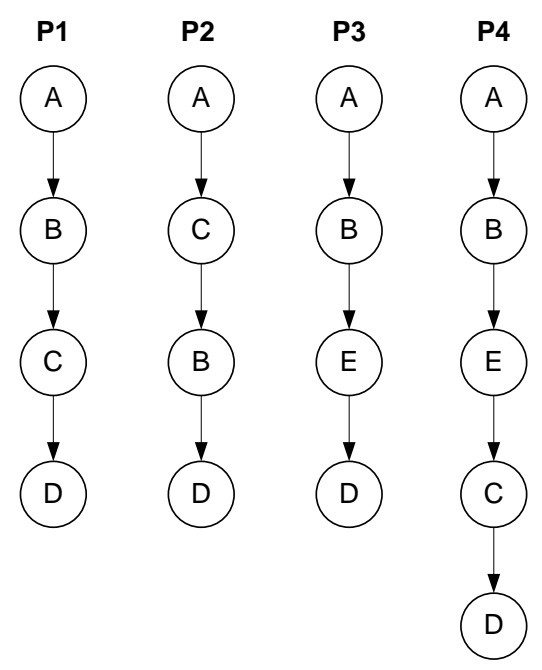

\section{Abbildung 3: Vier abstrakte Beispielprozesse zur Veranschaulichung der Methodik}

In Abbildung 3 sind vier Beispielprozesse P1, P2, P3 und P4 auf Basis der fünf Aktivitätstypen A, B, C, D und E dargestellt. In Tabelle 1 sind die dazugehörigen Codierungen auf Basis des Activity Similarity Measures aufgeführt. Es lässt sich erkennen, dass sich Prozesse P2, P3 und P4 auf Grund fachlicher Unterschiede in der Codierung (a) voneinander unterscheiden. Prozesse P1 und P2 allerdings weisen in dieser Codierung keine Unterschiede auf, weil die Reihenfolge bei der Codierung (a) nicht berücksichtigt wird. 


\section{Tabelle 1: Activity Similarity Measure (a), Codierung der Prozesse aus}

Abbildung 3

\begin{tabular}{|c|c|c|c|c|c|}
\hline \multirow{2}{*}{\multicolumn{2}{|c|}{ Aktivitätstyp }} & P1 & $\mathrm{P} 2$ & P3 & P4 \\
\hline & & & & & \\
\hline A & & 1 & 1 & 1 & 1 \\
\hline B & & 1 & 1 & 1 & 1 \\
\hline $\mathrm{C}$ & & 1 & 1 & 0 & 1 \\
\hline $\mathrm{D}$ & & 1 & 1 & 1 & 1 \\
\hline $\mathrm{E}$ & & 0 & 0 & 1 & 1 \\
\hline
\end{tabular}

Offensichtlich reicht eine Berücksichtigung des Vorhandenseins bzw. Nichtvorhandenseins einzelner Aktivitätstypen nicht aus, um die Struktur eines Prozesses in der notwendigen Genauigkeit zu erfassen. Daher berücksichtigen wir zusätzlich die direkten Nachfolger der Aktivitätstypen, Codierung (b). Dazu ist in Tabelle 2 exemplarisch die Transitionsmatrix von Prozess P1 und P4 dargestellt. Für den Prozess P1 ist erkennbar, dass Aktivitätstyp B direkter Nachfolger von Aktivitätstyp A ist, Aktivitätstyp C von B sowie Aktivitätstyp D von C. Die Unterschiede zwischen den Prozessen P1, P2 und P3 kommen in dieser Codierung gut zur Geltung. Die Unterschiede zwischen den Prozess P1 und P2 sind allerdings relativ groß, wenn nur die direkten Nachfolger betrachtet werden: Nach Codierung (b) unterschieden sich Prozesse P1 und P4 durch die Vorgänger-Nachfolger-Beziehungen (B,C) und (B,D), die in Prozess P1 vorhanden sind, aber nicht in Prozess P4 sowie durch (B,E), (E,C) und (C,D), welche in P4 vorhanden sind, nicht aber in P1. Der tatsächliche Unterschied zwischen beiden Prozessen ist allerdings nicht so groß: P4 unterscheidet sich von P1 nur dadurch, dass Aktivitätstyp E zusätzlich in P4 eingefügt ist.

\section{Tabelle 2: Transition Similarity Measure (b) für Prozesse P1 und P4 aus Abbildung 3, dargestellt als Matrix}

Prozess P1

\begin{tabular}{|c|l|l|l|l|l|}
\hline Aktivitätstyp & A & B & C & D & E \\
\hline A & 0 & 1 & 0 & 0 & 0 \\
\hline B & 0 & 0 & 1 & 0 & 0 \\
\hline C & 0 & 0 & 0 & 1 & 0 \\
\hline D & 0 & 0 & 0 & 0 & 0 \\
\hline E & 0 & 0 & 0 & 0 & 0 \\
\hline
\end{tabular}

Prozess P4

\begin{tabular}{|c|l|l|l|l|l|}
\hline Aktivitätstyp & A & B & C & D & E \\
\hline A & 0 & 1 & 0 & 0 & 0 \\
\hline B & 0 & 0 & 0 & 0 & 1 \\
\hline C & 0 & 0 & 0 & 1 & 0 \\
\hline D & 0 & 0 & 0 & 0 & 0 \\
\hline E & 0 & 0 & 1 & 0 & 0 \\
\hline
\end{tabular}

Daher berücksichtigen wir als dritte Codierung die transitive Hülle des Prozesses (c*). Bei der Transitiven Hülle werden nicht nur direkte, sondern auch alle indirekten Nachfolger berücksichtigt. Auf diese Weise fällt der Unterschied zwischen P1 und P4 nicht so stark ins Gewicht. Zur Illustration ist exemplarisch die Transitive Hülle von Prozess P1 in Tabelle 3 dargestellt. Es wird deutlich, dass Aktivitätstypen B, C und D direkte oder indirekte Nachfolger von A sind, C und D von B sowie D von C. Im Fall von Zyklen steht in der Diagonalen der transitiven Hülle eine 1. 


\section{Tabelle 3: Transitive Hülle ( $\left.c^{*}\right)$ für Prozesse P1 und P4 aus Abbildung 3, dargestellt als Matrix}

Prozess P1

\begin{tabular}{|c|l|l|l|l|l|}
\hline Aktivitätstyp & A & B & C & D & E \\
\hline A & 0 & 1 & 1 & 1 & 0 \\
\hline B & 0 & 0 & 1 & 1 & 0 \\
\hline C & 0 & 0 & 0 & 1 & 0 \\
\hline D & 0 & 0 & 0 & 0 & 0 \\
\hline E & 0 & 0 & 0 & 0 & 0 \\
\hline
\end{tabular}

Prozess P4

\begin{tabular}{|c|l|l|l|l|l|}
\hline Aktivitätstyp & A & B & C & D & E \\
\hline A & 0 & 1 & 1 & 1 & 1 \\
\hline B & 0 & 0 & 1 & 1 & 1 \\
\hline C & 0 & 0 & 0 & 1 & 0 \\
\hline D & 0 & 0 & 0 & 0 & 0 \\
\hline E & 0 & 0 & 1 & 1 & 0 \\
\hline
\end{tabular}

\section{Ähnlichkeitsmaß}

Zur Ähnlichkeitsmessung auf Basis der drei Codierungen haben wir übliche Ähnlichkeitsmaße aus der Statistik daraufhin überprüft, ob sie Ähnlichkeiten zwischen den Prozessen in geeigneter Weise verdichten. Die Evaluation verschiedener Ähnlichkeitsmaße ergab, dass die Wahl des Ähnlichkeitsmaßes keinen grundlegenden Einfluss auf die Ergebnisse zeigte. Für die weiteren Ausführungen liegt daher die Kosinusähnlichkeit ${ }^{1}$ zugrunde, die häufig für Ähnlichkeitsmessungen verwendet wird.

Zur Illustration haben wir die Ähnlichkeiten der Beispielprozesse aus Abbildung 3 unter Nutzung der Kosinusähnlichkeit in Ähnlichkeitsmatrizen dargestellt, siehe Tabelle 4. Ähnlichkeitsmatrizen sind symmetrisch, weil die Ähnlichkeit von Px zu Py den gleichen Wert hat wie die Ähnlichkeit von Py zu Px. Die Werte in der Hauptdiagonalen sind stets 1, da die Prozesse mit sich selbst stets vollständig identisch sind.

Die Beispiele machen deutlich, dass Ähnlichkeiten zwischen Prozessen auf Basis des Activity Similarity Measures (a) sehr hoch ausfallen. Prozesse P1 und P2 werden beispielsweise als vollkommen gleich aufgefasst, weil sie dieselben Aktivitätstypen verwenden. Das Transition Similarity Measure (b) hingegen bewertet die Ähnlichkeiten zwischen den Prozessen mit sehr geringen Werten. Geringe Abweichungen in der Reihenfolge der Aktivitäten wirken sich bereits stark auf die Ähnlichkeit aus. Die Prozesse P1 und P2 haben z. B. eine Ähnlichkeit von 0, da sie keine gemeinsame Vorgänger-Nachfolgerbeziehung haben. Die Ähnlichkeitsmatrix auf Basis der Transitiven Hülle ( $\left.\mathrm{c}^{*}\right)$ enthält mittelhohe Werte. Am Beispiel der Ähnlichkeit zwischen den Prozessen P1 und P2 wird deutlich, dass einerseits im Gegensatz zum Activity Similarity Measure (a) zwar Unterschiede zwischen beiden Prozessen festgestellt werden, dass andererseits aber diese Unterschiede nicht zu einer starken Unähnlichkeit führen, wie es beim Transition Similarity Measure (b) der Fall ist.

\section{Tabelle 4: Ähnlichkeitsmatrizen für die Beispielprozesse aus Abbildung 3 mit dem Kosinusähnlichkeitsmaß}

\begin{tabular}{|l|l|l|l|l|}
\hline & P1 & P2 & P3 & P4 \\
\hline P1 & 1.00 & 1.00 & 0.75 & 0.89 \\
\hline P2 & 1.00 & 1.00 & 0.75 & 0.89 \\
\hline P3 & 0.75 & 0.75 & 1.00 & 0.89 \\
\hline P4 & 0.89 & 0.89 & 0.89 & 1.00 \\
\hline
\end{tabular}

Ähnlichkeitsmatrix basierend auf dem Activity Similarity Measure (a)

\begin{tabular}{|l|l|l|l|l|}
\hline & P1 & P2 & P3 & P4 \\
\hline P1 & 1.00 & 0.00 & 0.33 & 0.58 \\
\hline P2 & 0.00 & 1.00 & 0.00 & 0.00 \\
\hline P3 & 0.33 & 0.00 & 1.00 & 0.58 \\
\hline P4 & 0.58 & 0.00 & 0.58 & 1.00 \\
\hline
\end{tabular}

Ähnlichkeitsmatrix basierend auf dem Transition Similarity Measure (b)

\begin{tabular}{|l|l|l|l|l|}
\hline & P1 & P2 & P3 & P4 \\
\hline P1 & 1.00 & 0.83 & 0.50 & 0.77 \\
\hline P2 & 0.83 & 1.00 & 0.50 & 0.65 \\
\hline P3 & 0.50 & 0.50 & 1.00 & 0.77 \\
\hline P4 & 0.77 & 0.65 & 0.77 & 1.00 \\
\hline
\end{tabular}

Ähnlichkeitsmatrix basierend auf der Transitiven Hülle (c*)

\section{Verknüpfung der Ähnlichkeitsmaße}

Der Empfehlung von Jung und Bae (2006) folgend, wurden die einzelnen Codierungen durch eine Konvexkombination miteinander gemischt, wobei $\alpha, \beta \in[0 ; 1]$ (mit $\alpha+\beta \in[0 ; 1]$ ) geeignet zu wählende Mischfaktoren sind:

\footnotetext{
${ }^{1}$ Die Kosinusähnlichkeit definiert zwei Vektoren als völlig unähnlich (Ähnlichkeit $=0$ ), wenn sie orthogonal zueinander stehen, und als völlig ähnlich (Ähnlichkeit = 1), wenn die Vektoren parallel zueinander liegen.
} 
Dabei sind

$$
\operatorname{sim}\left(P_{i}, P_{j}\right)=\alpha \cdot \operatorname{sim}_{a}\left(P_{i}, P_{j}\right)+\beta \cdot \operatorname{sim}_{b}\left(P_{i}, P_{j}\right)+(1-\alpha-\beta) \cdot \operatorname{sim}_{c *}\left(P_{i}, P_{j}\right)
$$

$P_{i}$ :Prozess $i$

\author{
$\operatorname{sim}\left(P_{i}, P_{j}\right):$ Ähnlichkeit zwischen den Prozessen $P_{i}$ und $P_{j}$ \\ $\operatorname{sim}_{x}$ : Ähnlichkeit auf Basis des Ähnlichkeitsmaßes $x$ \\ $a$ : Activity Similarity Measure \\ $b$ : Transition Similarity Measure \\ $c *$ : Ähnlichkeit auf Basis der Transitiven Hülle
}

Der Parameter $\beta$ bestimmt, wie stark die direkte Nachfolgebeziehung in die Ähnlichkeitsbetrachtungen einfließen soll. Durch Berücksichtigung der direkten Nachfolgebeziehung kann die beim Ähnlichkeitsmaß auf Basis der Transitiven Hülle (c*) im Gegensatz zur der Codierung (c) von Jung \& Bae fehlende Gewichtung zwischen direktem Nachfolger und weiteren Nachfolgern abgebildet werden.

Es hat sich in unserem konkreten Fall gezeigt, dass die Wahl der Parameter $\alpha$ und $\beta$ nur geringen Einfluss auf die Clusterung nimmt. Im Wesentlichen verändert sich die Größe der Cluster, die Zentren aber bleiben stabil. Da die Durchführung der Clusteranalyse performant und vollständig automatisiert durchführbar ist, konnten wir zahlreiche Versuche mit verschiedenen Parametern durchführen. Daraus ergab sich, dass die Clusteranalyse zu besonders guten Ergebnissen führt, wenn der Faktor für das Activity Similarity Measure (a, Faktor $\alpha$ ) und der für die Ähnlichkeit auf Basis der Transitiven Hülle (c*, Faktor $1-\alpha-\beta)$ etwa gleichgroß sind und für den Faktor für das Transition Similarity Measure (b, Faktor $\beta$ ) ein kleiner Wert (z. B. 0,1) gewählt wird. Die guten Ergebnisse bei einer geringen Gewichtung des Transition Similarity Measures (b) lassen sich dadurch erklären, dass dieses Ähnlichkeitsmaß zu großen Unähnlichkeiten bei bereits kleinen Unterschieden zwischen Prozessen führt (vgl. die Diskussion oben der Ähnlichkeitsmatrizen zu den Beispielprozessen).

\title{
Clusteralgorithmus
}

Als Clusteralgorithmus haben wir mehrere Algorithmen evaluiert und uns letztlich für den PAM-Algorithmus (Partitioning Around Medoids) entschieden, der gute Clusterergebnisse lieferte. Als Referenzprozess haben wir denjenigen Prozess definiert, dessen Abstand zum Clusterzentrum am geringsten ist.

\section{Beispielprozess Personenneuanlage}

Die Analyse haben wir auf den anonymisierten Daten von 371 Instituten am Beispielprozess Personenneuanlage durchgeführt. Insgesamt waren zum Zeitpunkt der Auswertung 581 Prozessvarianten bei den Instituten aktiv im Einsatz. Dabei konnten wir vier Cluster identifizieren. In

Abbildung 4 sind die Aktivitätstypen der Referenzprozesse aus den vier Clustern in einer Übersicht dargestellt. Die technischen Aktivitätstypen Geschäftsprozess starten und Geschäftsprozess beenden sowie der Aktivitätstyp Person anlegen kommen in jedem Prozess vor. Die Zentren des ersten und des dritten Clusters sind mittelgroße Prozesse, in denen eine bestimmte Auswahl an Aktivitätstypen getroffen worden ist. Das Zentrum des vierten Clusters ist ein Prozess, der nur eine einzige fachliche Aktivität auf Basis des Aktivitätstypen Person anlegen beinhaltet und somit den kleinstmöglichen Prozess darstellt. Das Zentrum des zweiten Clusters hingegen stellt einen sehr umfassenden Prozess dar, welcher die Möglichkeit bietet, eine Vielzahl an Informationen zu einer neuen Person aufzunehmen und auch Spezialfälle abzudecken.

Das Zentrum von Cluster 1 ist der Prozess, der aktuell als Standardprozess von den Verbänden definiert ist und durch die Finanz Informatik bereitgestellt wird. In diesem Cluster finden daher diejenigen Institute ihre Prozesse, die mit dem ausgelieferten Standard weitgehend zufrieden waren. In Cluster 3 finden sich Prozessvarianten von Instituten, die kleinere Anpassungen an dem Standardprozess durchgeführt haben. Das Zentrum von Cluster zwei, ein aus fachlicher Sicht ausführlicher Prozess, ist in einem früheren Release als Standardprozess ausgeliefert worden; offenbar waren einige Institute mit diesem früheren Prozess zufrieden und wollen ihn weiterverwenden. Cluster 4, der kleinste Cluster, enthält letztlich den minimalen Prozess, der die geringste Durchlaufzeit und die geringsten IT-Kosten abbildet.

An der Vielfalt der eingesetzten Prozesse lässt sich erkennen, dass die einzelnen Institute unterschiedliche Optimalitätskriterien für ihre Prozesse haben. Während die Prozesse in Cluster vier, isoliert betrachtet, die Prozesskosten des Prozesses Personenneuanlage minimieren, verfolgen die Institute in Cluster zwei ein anderes Ziel: Durch die möglichst frühzeitige Aufnahme vieler, möglicherweise relevanter Kundendaten werden in nachfolgenden Prozessen Zeit und Kosten eingespart. 
Beispielsweise holen sich einige Institute durch die Aufnahme von Werbekontaktdaten bei der Anlage einer neuen Person die Erlaubnis ein, dem Kunden Werbepost zuschicken zu dürfen. Wenn eine solche Erlaubnis erst zu einem anderen Zeitpunkt eingeholt wird, kann dies mit großem Aufwand verbunden sein. Weiterhin verwenden einige Institute den empfohlenen Standardprozess, was darauf hindeutet, dass der Standardprozess die Anforderungen dieser Institute abdeckt und der eventuell vorhandene individuelle Anpassungsbedarf den Aufwand für eine individuelle Prozessadministration nicht rechtfertigt.

Aber auch innerhalb der jeweiligen Häuser werden offensichtlich unterschiedliche Prozessvarianten eingesetzt, da die Gesamtzahl der Prozessvarianten mit 581 deutlich größer als die Anzahl der Institute (371) ist. Personenanlagen werden nicht nur im FrontOffice mit dem Kunden durchgeführt, sondern auch im Back-Office. Eine Personenneuanlage, bei der der Kunde vor Ort ist, erfordert fachlich ggf. einen anderen Ablauf als im Back-Office, wenn der Kunde nicht vor Ort ist. Im ersten Fall können weitere Daten einfach beim Kunden erfragt und erfasst werden.

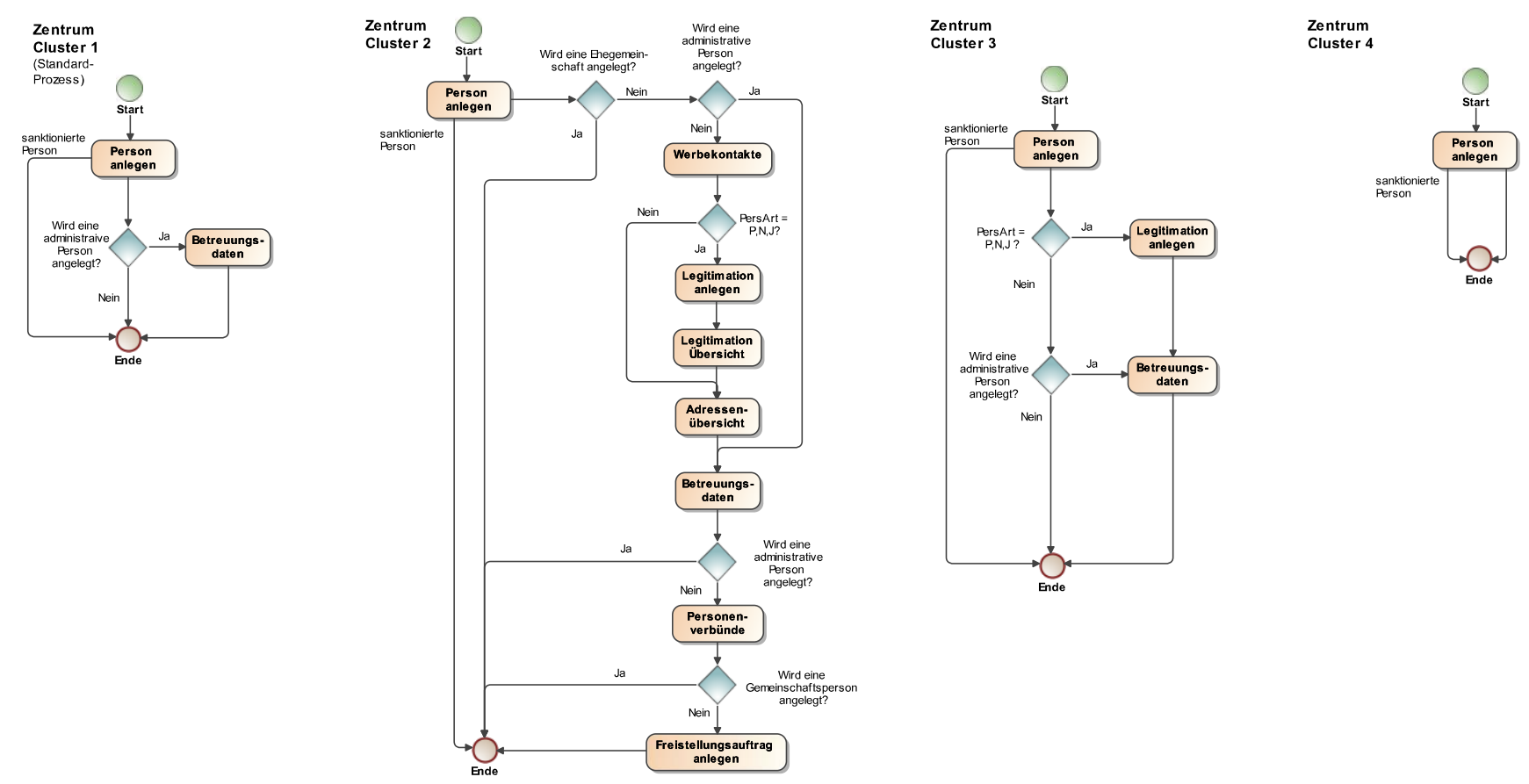

\section{Abbildung 4: Zentren der vier Cluster als Ablaufdiagramme}

\section{Diskussion des entwickelten Verfahrens für das Sparkassen-Umfeld}

\section{Nutzenpotenzial}

Für die Regionalverbände und die Institute ist das entwickelte Verfahren von großem Nutzen. Durch die empirische Referenzmodellermittlung können die Bedürfnisse der einzelnen Institute mit überschaubarem Aufwand analysiert werden und Vorschläge für die Anpassung des Standardprozesses abgeleitet werden. Da das Verfahren deskriptiv ist, müssen die fachlichen Anforderungen der einzelnen Institute nicht aufwändig ermittelt werden. Insbesondere normative Diskussionen um geeignete Optimierungskriterien für Geschäftsprozesse müssen nicht mit den einzelnen Instituten geführt werden.

Um das Nutzenpotenzial bei den Instituten zu ermitteln, haben wir Interviews mit über zwanzig Vertretern von Instituten durchgeführt, die für die Organisation oder die IT ihres jeweiligen Hauses verantwortlich oder mitverantwortlich sind. Grundsätzlich begrüßen die Interviewpartner das Vorgehen aus mehreren Gründen: Es ist Konsens unter den Befragten, dass die empirisch ermittelten Referenzprozesse eine große Praxisnähe aufweisen. Die Befragten äußerten daher, dass sie die empirisch ermittelten Referenzprozesse gegenüber solchen Referenzprozessen vorziehen, die aus rein fachlichen Überlegungen heraus konstruiert wurden. Darüber hinaus begrüßen sie die Möglichkeit, aus mehreren Referenzprozessen einen für sie passenden aussuchen zu können. Aufgrund des geringeren administrativen Aufwands für die einzelnen Institute würden fast alle der befragten Institute zu einem dieser Referenzprozesse migrieren, insbesondere dann, wenn zu den Standardprozessen weitere 
Servicedienstleistungen durch die Finanz Informatik angeboten werden. Da die Institute dann wieder Standardprozesse der Finanz Informatik mit den entsprechenden Servicevereinbarungen nutzen, werden sich die Administrationsaufwände bei neuen gesetzlichen oder fachlichen Anforderungen dauerhaft verringern. Weiterhin besteht ein großes Interesse, von anderen Instituten zu lernen und Anregungen für die eigenen Prozesse zu sammeln. Auch zu diesem Zweck wird das Verfahren als sehr gut geeignet bewertet.

Zusammenfassend wird das richtige Zusammenspiel von vorgelagerter Prozessmodellierung und nachgelagerter empirischer Ermittlung von Referenzprozessen dauerhaft zu einer größeren Akzeptanz der bereitgestellten Geschäftsprozesse durch die Institute und zu einer Verbesserung der Praxisnähe der bereitgestellten Standardprozesse führen.

\section{Limitationen und nächste Schritte}

In den Interviews mit den Vertretern der Institute wurde neben den beschriebenen Vorteilen auch deutlich, dass es sich um ein rückwärts gerichtetes Verfahren handelt, das erst im Nachhinein greift. Für Anpassungen an neue Rahmenbedingungen, z. B. an neue gesetzliche Vorgaben, ist es auch weiterhin notwendig, von fachlichen Anforderungen auszugehen. Hier ist die gestalterische und analytische Vorarbeit des Deutschen Sparkassen- und Giroverbands sowie der Regionalverbände unerlässlich. Damit das hier vorgestellte vergangenheitsorientierte Verfahren zu brauchbaren Ergebnissen führt, ist es wichtig, dass zunächst die einzelnen Institute im Rahmen des Praxiseinsatzes den ausgelieferten Standardprozess an ihre individuellen Anforderungen anpassen, falls sie dies für erforderlich halten. Erst im Anschluss kann die empirische Analyse brauchbare Ergebnisse erzielen. Es ist darauf zu achten, dass bei der Etablierung des Verfahrens keine reine Konsummentalität seitens der Institute entsteht. Diese Gefahr ist jedoch nach derzeitiger Einschätzung als nicht sehr hoch zu bewerten, da die Institute die Prozessanpassungen fachlich getrieben auf Grund ihrer internen Strukturen umsetzen. Insbesondere nehmen die Institute, die eigene Prozessvarianten einsetzen, entscheidenden Einfluss auf die Entwicklung des angepassten Standardprozesses und minimieren damit dauerhaft die eigenen Administrationsaufwände, so dass sich der Aufwand für die Anpassung des Prozesses auch dauerhaft für die Institute lohnt.

Mit dem entwickelten Verfahren können Referenzprozesse im OSPlus empirisch ermittelt werden. Das Verfahren wurde auf den Prozess Personenneuanlage angewendet, um den Nachweis der Praxistauglichkeit zu erbringen. Es ist geplant, diese Analyse auch mit weiteren Prozessen durchzuführen. In jedem Fall werden die Ergebnisse der jeweiligen Analyse den zuständigen Regionalverbänden und Fachgremien vorgelegt. Dort wird dann bewertet, ob und in welcher Form die empirisch ermittelten Referenzprozesse in die Weiterentwicklung einfließen werden. Eine Bereitstellung der ermittelten Referenzprozesse erfolgt erst nach der erfolgten Bewertung. Anschließend können die empirisch ermittelten Referenzprozesse als neue Standardprozesse an die Institute ausgeliefert werden. In einer weiterführenden Untersuchung können dann die Migrationsbewegungen der einzelnen Institute hin zu den neuen Standardprozessen beobachtet und analysiert werden.

\section{Lessons Learned}

In dem hier beschriebenen Projekt wurden stark die Rahmenbedingungen innerhalb der Sparkassen-Finanzgruppe und die des Produktes OSPlus berücksichtigt. Besonders förderlich für die Clusteranalyse ist die Tatsache, dass alle Prozesse bei allen Instituten auf denselben Aktivitätstypen basieren und die Prozessmodelle in auswertbarer Form vorliegen. Nur so ist es möglich, die Ähnlichkeitsberechnungen durchzuführen. Weiterhin ist es sehr günstig, dass eine hohe Zahl an Prozessvarianten vorliegt. Das hier vorgestellte Verfahren ist nur dann auf weitere Umgebungen generalisierbar, wenn dort vergleichbare Rahmenbedingungen vorliegen.

Neben dem vorgestellten Verfahren lassen sich als Ergebnis des Projektes auch Aussagen zur Akzeptanz von Referenzmodellen treffen. Es wurde deutlich, dass ein wichtiges Kriterium für die Akzeptanz der Referenzmodelle ihre Praxisnähe ist. Von vielen Interviewpartnern wurde geäußert, dass sie empirisch ermittelte Referenzprozesse für praxisnäher halten als solche, die im Vorhinein vorwiegend aus theoretischen Überlegungen, gesetzlichen Anforderungen und individueller Erfahrung entstehen.

\section{Literatur}

Delfmann, P. (2006). Adaptive Referenzmodellierung: Methodische Konzepte zur Konstruktion und Anwendung wiederverwendungsorientierter Informationsmodelle. Westfälische Wilhelms-Universität Münster, Münster.

Jung, J.-Y. \& Bae, J. (2006). Workflow Clustering Method Based on Process Similarity. In: Computational Science and Its Applications - ICCSA 2006 (Gavrilova, M. et al., Eds), Springer, Berlin.

Winter, R. (2011). Problem Analysis for Situational Artefact Construction in Information Systems. In: A Tribute to Marco de Marco, Springer, Berlin. 
Autoren

Prof. Dr. Stephan Aier

ist Assistenzprofessor für Informationsmanagement an der School of Management der Universität St.Gallen (HSG). Am dortigen Institut für Wirtschaftsinformatik (IWI-HSG) leitet er am Lehrstuhl von Prof. Dr. Robert Winter den Arbeitsbereich Architekturund Integrationsmanagement.

\section{Dr. Michael Fichter}

ist als Abteilungsleiter bei der Finanz Informatik GmbH \& Co. KG verantwortlich für die Entwicklung und Bereitstellung der Geschäftsprozesssteuerung. Er unterstützt die Entwicklungseinheiten der Finanz Informatik bei der Umsetzung von Geschäftsprozessen auf Basis der Geschäftsprozesssteuerung und stellt Auswertungsmöglichkeiten zur Prozessoptimierung und Standardisierung von Geschäftsprozessen zur Verfügung.

\section{Christian Fischer, MScIS}

ist Doktorand bei Prof. Dr. Robert Winter am Institut für Wirtschaftsinformatik der Universität St.Gallen (HSG). Er forscht im Arbeitsbereich Architektur- und Integrationsmanagement mit den Schwerpunkten Geschäftsprozessmanagement, Design Science Research und Innovationsmanagement. 